\title{
Extremal Problems for Subset Divisors
}

\author{
Tony Huynh* \\ Department of Computer Science \\ University of Rome \\ Rome, Italy \\ tony.bourbaki@gmail.com
}

Submitted: Jun 5, 2013; Accepted: Feb 20, 2014; Published: Feb 28, 2014

Mathematics Subject Classifications: 05A15, 05D05

\begin{abstract}
Let $A$ be a set of $n$ positive integers. We say that a subset $B$ of $A$ is a divisor of $A$, if the sum of the elements in $B$ divides the sum of the elements in $A$. We are interested in the following extremal problem. For each $n$, what is the maximum number of divisors a set of $n$ positive integers can have? We determine this function exactly for all values of $n$. Moreover, for each $n$ we characterize all sets that achieve the maximum. We also prove results for the $k$-subset analogue of our problem. For this variant, we determine the function exactly in the special case that $n=2 k$. We also characterize all sets that achieve this bound when $n=2 k$.
\end{abstract}

Keywords: extremal combinatorics; exact enumeration

\section{Introduction}

Let $A$ be a finite set of positive integers and let $B$ be a subset of $A$. We say that $B$ is a divisor of $A$, if the sum of the elements in $B$ divides the sum of the elements in $A$. We are interested in the number of divisors a set of positive integers can have. Toward that end, we let $d(A)$ be the number of divisors of $A$ and we let $d(n)$ be the maximum value of $d(A)$ over all sets $A$ of $n$ positive integers. We also study the $k$-subset version of this problem. That is, we define $d_{k}(A)$ to be the number of $k$-subset divisors of $A$ and we let $d(k, n)$ be the maximum value of $d_{k}(A)$ over all sets $A$ of $n$ positive integers.

This work is motivated by Problem 1 from the 2011 International Mathematical Olympiad [1], where it is asked to determine $d(2,4)$.

*Supported by the European Research Council under the European Unions Seventh Framework Programme (FP7/2007-2013)/ERC Grant Agreement no. 279558. 
Problem 1. Determine $d(2,4)$. Moreover, find all sets of four positive integers $A$ with exactly $d(2,4) 2$-subset divisors.

We begin by presenting the solution to Problem 1 .

Lemma 2. For any set $A$ of four positive integers, $d_{2}(A) \leqslant 4$. Moreover, $d_{2}(A)=4$ if and only if

$$
A=\{a, 5 a, 7 a, 11 a\} \text { or } A=\{a, 11 a, 19 a, 29 a\}
$$

for some $a \in \mathbb{N}$.

Proof. Let $A=\left\{a_{1}, a_{2}, a_{3}, a_{4}\right\}$ be a set of positive integers with $a_{1}<a_{2}<a_{3}<a_{4}$. We use $\sum A$ to denote the sum of the elements in $A$. Note that $\frac{1}{2} \sum A<a_{2}+a_{4}<a_{3}+a_{4}<\sum A$. Thus $d_{2}(A) \leqslant 4$ as neither $\left\{a_{2}, a_{4}\right\}$ nor $\left\{a_{3}, a_{4}\right\}$ can divide $A$.

Suppose $d_{2}(A)=4$. This implies that both $\left\{a_{1}, a_{4}\right\}$ and $\left\{a_{2}, a_{3}\right\}$ divide $A$, and hence $a_{1}+a_{4}=a_{2}+a_{3}$. Since $\left\{a_{1}, a_{2}\right\}$ and $\left\{a_{1}, a_{3}\right\}$ also divide $A,\left(a_{1}+a_{2}\right) \mid\left(a_{3}+a_{4}\right)$ and $\left(a_{1}+a_{3}\right) \mid\left(a_{2}+a_{4}\right)$. Therefore, there exist $2 \leqslant j<k$ such that

(i) $a_{1}+a_{4}=a_{3}+a_{2}$,

(ii) $j\left(a_{1}+a_{3}\right)=a_{2}+a_{4}$, and

(iii) $k\left(a_{1}+a_{2}\right)=a_{3}+a_{4}$.

Adding $(i)$ and $(i i)$, we obtain $(j+1) a_{1}+(j-1) a_{3}=2 a_{2}$. Since $a_{3}>a_{2}$, it follows that $j=2$. Substituting $j=2$ and taking $3(i)+2(i i)+($ iii $)$ we obtain $(k+7) a_{1}=(5-k) a_{2}$. This implies $(5-k)>0$, and so $k \in\{3,4\}$. By solving the systems corresponding to the values $k=3$ and $k=4$ we are lead to the respective solutions

$$
A=\{a, 5 a, 7 a, 11 a\} \text { and } A=\{a, 11 a, 19 a, 29 a\} .
$$

It is easy to check that any set $A$ of the above form does indeed satisfy $d_{2}(A)=4$.

\section{Lower bounds for $d(n)$ and $d(k, n)$}

In this section, we give constructions for sets of positive integers with many divisors and many $k$-subset divisors. In the next section we derive matching upper bounds for $d(n)$ and $d(n, 2 n)$ and hence these sets are optimal. Moreover, in Section 4, we will show that these are almost all the sets achieving the maximum values.

Recall that $d(n)$ (respectively, $d(k, n)$ ) is the maximum number of divisors (respectively, $k$-subset divisors) a set of $n$ positive integers can have. By convention, the sum of the elements in the empty set is zero, and so the empty set does not divide any set (except itself).

Lemma 3. For all $n \geqslant 1, d(n) \geqslant 2^{n-1}$. 
Proof. The lemma clearly holds if $n=1$. Thus, assume $n \geqslant 2$ and let $A^{\prime}$ be any set of $n-1$ positive integers. We show that we can choose an element $a$ such that $A^{\prime} \cup\{a\}$ has $2^{n-1}$ divisors. Let

$$
S:=\left\{s \in \mathbb{N}: s=\sum B \text { for some non-empty } B \subseteq A^{\prime}\right\} .
$$

Let $\ell$ be the least common multiple of the elements in $S$, and let $\ell^{\prime}$ be a multiple of $\ell$ such that $\ell^{\prime}-\sum A^{\prime} \notin A$. Set $a:=\ell^{\prime}-\sum A^{\prime}$ and consider $A:=A^{\prime} \cup\{a\}$. Note that $\sum A=\ell^{\prime}$. Therefore, every non-empty subset of $A^{\prime}$ divides $A$. Also, $A$ divides $A$. Thus $d(A) \geqslant 2^{n-1}$, as required.

A similar construction also gives lower bounds for $d(k, n)$.

Lemma 4. For all $k, n \geqslant 1, d(k, n) \geqslant\left(\begin{array}{c}n-1 \\ k\end{array}\right)$.

Proof. Again, the lemma clearly holds for $n=1$. So, for $n \geqslant 2$ arbitrarily choose a set $A^{\prime}$ of $n-1$ positive integers and let

$$
S:=\left\{s \in \mathbb{N}: s=\sum B \text { for some } B \subseteq A^{\prime} \text { with }|B|=k\right\} .
$$

The rest of the proof is identical to the proof of the previous lemma. That is, we construct $a$ such that all $k$-subsets of $A^{\prime}$ divide $A^{\prime} \cup\{a\}$.

We point out that the same technique shows that the corresponding minimization problems for $d(A)$ and $d_{k}(A)$ are easy. Namely, define $A$ to be prime if the only divisor of $A$ is $A$ itself.

Claim 5. For each $n \in \mathbb{N}$, there exists infinitely many prime $n$-sets of integers.

Proof. Arbitrary choose a set $A^{\prime}$ of $n-1$ positive integers, with $1 \notin A^{\prime}$. Choose a prime number $p$ such that $p \geqslant 2 \sum A^{\prime}$. Finish by setting $a:=p-\sum A^{\prime}$ and $A:=A^{\prime} \cup\{a\}$.

\section{$3 \quad$ Upper bounds for $d(n)$ and $d(n, 2 n)$}

Let $A$ be a set of positive integers. We say that a subset $B$ of $A$ is a halving set if $\sum B=\frac{1}{2} \sum A$. Evidently, $B$ is a halving set if and only if $A \backslash B$ is a halving set. The next lemma is also obvious, but quite useful.

Lemma 6. If $B$ and $C$ are distinct halving sets, then $|B \triangle C|>2$.

A separation of $A$ is a pair $\{B, C\}$, where $B$ and $C$ are disjoint subsets of $A$ with $B \cup C=A$. Note that $\{B, C\}=\{C, B\}$. A strong separation is a separation $\{B, C\}$ where $|B|=|C|$. We say that $\{B, C\}$ is barren if neither $B$ nor $C$ divides $A$, neutral if exactly one of $B$ or $C$ divides $A$, and abundant if both $B$ and $C$ divide $A$. Note that $\{B, C\}$ is an abundant separation if and only if $B$ and $C$ are both halving sets.

Thus, one approach to obtain upper bounds for $d(n)$ (respectively, $d(n, 2 n)$ ) is to bound the number of abundant separations (respectively, abundant strong separations) of $A$. 
Lemma 7. Let $A$ be a set of $n$ positive integers. Then $d(A) \leqslant 2^{n-1}+h$, where $h$ is the number of abundant separations of $A$.

Proof. Partition $2^{A}$ into pairs $\{B, A \backslash B\}$. There are $2^{n-1}$ such separations. Finish by observing that a separation contributes 0 to $d(A)$ if it barren, 1 to $d(A)$ if it is neutral, and 2 to $d(A)$ if it is abundant.

Similarly, we have the following lemma.

Lemma 8. Let $A$ be a set of $2 n$ positive integers. Then $d_{n}(A) \leqslant \frac{1}{2}\left(\begin{array}{c}2 n \\ n\end{array}\right)+h$, where $h$ is the number of abundant strong separations of $A$.

Note that these bounds match the lower bounds from the previous section if $h=0$. However, it is possible for a set to have many halving sets. For example, consider $A=$ $\{1, \ldots, 4 \ell\}$. A theorem of Stanley [13] shows that this example is in fact worst possible.

Fortunately, we are able to determine $d(n)$ and $d(n, 2 n)$ using a different approach.

We first handle $d(n)$ by showing that the bound from Lemma 3 is best possible for almost all values of $n$.

Lemma 9. For all $n \geqslant 4, d(n)=2^{n-1}$.

Proof. Let $n \geqslant 4$ and $A$ be a set of $n$ positive integers. By Lemma 3 it suffices to show $d(A) \leqslant 2^{n-1}$. If no separations of $A$ are abundant, then we are done by Lemma 7 . So we may assume that $A$ contains an abundant separation. We proceed by defining an injection $\phi$ from the set of abundant separations to the set of barren separations. Let $\{B, C\}$ be an abundant separation. We may assume that $\min A \in B$. Define $\phi(\{B, C\})$ to be $\{B \backslash \min A, C \cup \min A\}$. First note that $\phi$ is injective. Secondly, if $\min A<\frac{1}{6} \sum A$, then $\frac{1}{3} \sum A<\sum(B \backslash \min A)<\frac{1}{2} \sum A$. Thus, if min $A<\frac{1}{6} \sum A$, then $\phi$ maps abundant separations to barren separations. So we are done unless $\min A \geqslant \frac{1}{6} \sum A$.

Observe that if $A$ contains a halving set $H$ of size at least 3 , then $\min A \leqslant \min H<$ $\frac{1}{6} \sum A$. Therefore, we are done unless $n=4$. Let $A:=\left\{a_{1}, \ldots, a_{4}\right\}$ with $a_{1}<a_{2}<a_{3}<$ $a_{4}$. Since there are no halving sets of $A$ of size 3 , it follows that $\left\{\left\{a_{1}, a_{4}\right\},\left\{a_{2}, a_{3}\right\}\right\}$ is the unique abundant separation of $A$. Now, since $a_{1} \geqslant \frac{1}{6} \sum A$ it follows that $\frac{1}{3} \sum A<$ $a_{1}+a_{2}<\frac{1}{2} \sum A$. Thus, $\left\{\left\{a_{1}, a_{2}\right\},\left\{a_{3}, a_{4}\right\}\right\}$ is a barren separation, so we are done by defining $\phi\left(\left\{\left\{a_{1}, a_{4}\right\},\left\{a_{2}, a_{3}\right\}\right\}\right):=\left\{\left\{a_{1}, a_{2}\right\},\left\{a_{3}, a_{4}\right\}\right\}$.

It is easy to determine the small values of $d(n)$ by hand. We omit the details.

Lemma 10. We have $d(1)=1, d(2)=2$, and $d(3)=5$. If $|A|=3$, then $d(A)=5$ if and only if $A=\{a, 2 a, 3 a\}$ for some $a \in \mathbb{N}$.

We now show that for $d(n, 2 n)$, the lower bound from Lemma 4 is also best possible for $n \geqslant 3$.

Lemma 11. For all $n \geqslant 3, d(n, 2 n)=\frac{1}{2}\left(\begin{array}{c}2 n \\ n\end{array}\right)$. 
Proof. Let $A$ be a set of $2 n$ positive integers, with $n \geqslant 3$. By Lemma 4 , it suffices to show that $d_{n}(A) \leqslant \frac{1}{2}\left(\begin{array}{c}2 n \\ n\end{array}\right)$. First observe that if $A$ does not contain any abundant strong separations, then we are done by Lemma 8 .

So, we may assume that $A$ contains an abundant strong separation. In this case, we proceed by defining an injection $\phi$ from the family of abundant strong separations to the family of barren strong separations. Let $\{B, C\}$ be an abundant strong separation. We define

$$
\phi(\{B, C\}):=\{(B \backslash \min B) \cup \min C,(C \backslash \min C) \cup \min B\} .
$$

First note that if $\phi\left(\left\{B_{1}, C_{1}\right\}\right)=\phi\left(\left\{B_{2}, C_{2}\right\}\right)$ for $\left\{B_{1}, C_{1}\right\} \neq\left\{B_{2}, C_{2}\right\}$, then by relabelling we may assume that $\left|B_{1} \cap B_{2}\right|=n-1$. However, this contradicts Lemma 6 . So $\phi$ is indeed an injection. We finish the proof by showing that $\phi$ maps abundant separations to barren separations. We may assume that $\min B<\min C$. Let $B^{\prime}:=(B \backslash \min B) \cup \min C$ and $C^{\prime}:=(C \backslash \min C) \cup \min B$. Clearly, $B^{\prime}$ does not divide $A$. Also, as $\sum B=\sum C=$ $\frac{1}{2} \sum A$, both $\min C$ and $\min B$ are strictly less than $\frac{1}{2 n} \sum A$. Therefore

$$
\left(\frac{1}{2}-\frac{1}{2 n}\right) \sum A<\sum C^{\prime}<\frac{1}{2} \sum A .
$$

Since $n \geqslant 3,\left(\frac{1}{2}-\frac{1}{2 n}\right) \geqslant \frac{1}{3}$. Thus, $C^{\prime}$ also does not divide $A$.

\section{Characterizing all extremal sets}

We now characterize all subsets of integers that achieve the bounds in Lemma 9 and Lemma 11. Let $A:=\left\{a_{1}, \ldots, a_{n}\right\}$ be a set of $n$ positive integers with $a_{1}<\cdots<a_{n}$. We say that $A$ is an anti-pencil if the set of divisors of $A$ consists of all non-empty subsets of $A \backslash\left\{a_{n}\right\}$ together with $A$ itself. Similarly, $A$ is a $k$-anti-pencil if the set of $k$-subset divisors of $A$ is the set of all $k$-subsets of $A \backslash\left\{a_{n}\right\}$. Observe that the constructions in Section 2 completely describe the set of all anti-pencils and the set of all $k$-anti-pencils.

We will need the following two simple observations to aid with the case analysis.

Lemma 12. If $k, \ell$, and $m$ are positive integers such that $\frac{1}{k}+\frac{1}{\ell}=\frac{1}{m}$, then $k+\ell$ divides $k \ell$.

Lemma 13. If $k<\ell$ are positive integers such that $\frac{1}{k}+\frac{1}{\ell}=\frac{1}{2}$, then $k=3$ and $\ell=6$.

Here is our first characterization.

Lemma 14. For $n \geqslant 5$, a set of $n$ positive integers has exactly $2^{n-1}$ divisors if and only if it is an anti-pencil. A set of four positive integers has 8 divisors if and only if it is an anti-pencil or of the form $\{a, 2 a, 3 a, 6 a\}$ for some $a \in \mathbb{N}$.

Proof. One direction is obvious. For the other direction, let $n \geqslant 4$ and $A:=\left\{a_{1}, \ldots, a_{n}\right\}$ have exactly $2^{n-1}$ divisors. We may assume that $a_{n} \leqslant \frac{1}{2} \sum A$, else $A$ is an anti-pencil and we are done. 
We claim that $a_{2} \geqslant \frac{1}{6} \sum A$. Suppose not. Given an abundant separation of $A$, let $\phi_{1}$ be the map which moves $a_{1}$ across the separation and let $\phi_{2}$ be the map which moves $a_{2}$ across the separation. Since $a_{1}$ and $a_{2}$ are both less than $\frac{1}{6} \sum A$, we again have that $\phi_{1}$ and $\phi_{2}$ are injective maps from the set of abundant separations to the set of barren separations. Moveover, by Lemma 6, the images of $\phi_{1}$ and $\phi_{2}$ are disjoint. Therefore, $A$ has more barren separations than abundant separations, which is a contradiction.

We next claim that $A$ does not contain any abundant separations or $A=\{a, 2 a, 3 a, 6 a\}$ for some $a \in \mathbb{N}$. Suppose $\{B, C\}$ is an abundant separation. If $\max \{|B|,|C|\} \geqslant 4$ or $\min \{|B|,|C|\} \geqslant 3$, then $a_{2}<\frac{1}{6} \sum A$; a contradiction. In particular, this implies $n \in\{4,5\}$.

We first handle the case $n=5$. Let $B:=\left\{b_{1}, b_{2}\right\}$ and $C:=\left\{c_{1}, c_{2}, c_{3}\right\}$ with $b_{1}<b_{2}$ and $c_{1}<c_{2}<c_{3}$. By Lemma $6,\{B, C\}$ is the unique abundant separation of $A$. Now, if $a_{1}=b_{1}$, then $a_{2} \leqslant c_{1}<\frac{1}{6} \sum A$; a contradiction. Thus, $a_{1}=c_{1}$. It follows that $\left\{\left\{b_{1}, b_{2}, c_{1}\right\},\left\{c_{2}, c_{3}\right\}\right\}$ is the unique barren separation of $A$. In particular $\left\{a_{i}\right\}$ divides $A$ for all $i \in[5]$. Therefore, there exist positive integers $m_{1}>\cdots>m_{5}$ such that $m_{i} a_{i}=\sum A$. Since $m_{2} \leqslant 6$ and $m_{5} \geqslant 3$ we must have $m_{2}=6, m_{3}=5, m_{4}=4$, and $m_{5}=3$. Now choose a 2-subset $A^{\prime}$ of $\left\{a_{2}, a_{3}, a_{4}\right\}$ such that $A^{\prime} \neq\left\{c_{2}, c_{3}\right\}$. Since $\sum A^{\prime}<\frac{1}{2} \sum A$ and $A^{\prime} \neq\left\{c_{2}, c_{3}\right\}$, it follows that $A^{\prime}$ divides $A$. However, this is a contradiction, since the equation $\frac{1}{k}+\frac{1}{\ell}=\frac{1}{m}$ has no positive integer solutions for $\{k, \ell\} \subset\{4,5,6\}$ by Lemma 12 .

We thus have $n=4$. Again by Lemma $6,\{B, C\}$ is the unique abundant separation of $A$. Thus, there is a unique barren separation $\left\{B^{\prime}, C^{\prime}\right\}$ of $A$. First suppose $|B|=|C|=$ 2. By relabelling if necessary, $B=\left\{a_{1}, a_{4}\right\}$ and $C=\left\{a_{2}, a_{3}\right\}$. Since $\left\{B^{\prime}, C^{\prime}\right\}$ is the unique barren separation of $A$, at least three of $\left\{a_{1}\right\},\left\{a_{2}\right\},\left\{a_{3}\right\}$ or $\left\{a_{4}\right\}$ divide $A$. By the pigeonhole principle, both members of $B$ divide $A$ or both members of $C$ divide $A$. By Lemma 13, we either have $6 a_{1}=\sum A=3 a_{4}$ or $6 a_{2}=\sum A=3 a_{3}$. In the second case, swapping $a_{1}$ and $a_{2}$ or swapping $a_{3}$ and $a_{4}$ in $\{B, C\}$ both yield barren separations, which contradicts the uniqueness of $\left\{B^{\prime}, C^{\prime}\right\}$. The first case is also impossible as any single swap of $\{B, C\}$ yields a barren separation. Therefore, we may assume $B=\left\{a_{4}\right\}$ and $C=\left\{a_{1}, a_{2}, a_{3}\right\}$. Now consider the sets

$$
\left\{a_{2}\right\},\left\{a_{3}\right\},\left\{a_{1}, a_{2}\right\},\left\{a_{1}, a_{3}\right\},\left\{a_{2}, a_{3}\right\} \text {. }
$$

Since $A$ has exactly one barren separation, at least four of these sets divide $A$. Let $m_{1} \leqslant m_{2} \leqslant m_{3} \leqslant m_{4}$ be the multiples that appear for these four divisors $D_{1}, \ldots, D_{4}$. Note that $m_{4} \leqslant 6$ since $a_{2} \geqslant \frac{1}{6} \sum A$. Suppose these multiples are all distinct. In this case, it follows that $m_{1}=3, m_{2}=4, m_{3}=5, m_{4}=6$. Since $\frac{1}{3}+\frac{1}{6}=\frac{1}{2}, D_{1}$ and $D_{4}$ must partition $\left\{a_{1}, a_{2}, a_{3}\right\}$. It cannot be that $\left\{D_{1}, D_{4}\right\}=\left\{\left\{a_{3}\right\},\left\{a_{1}, a_{2}\right\}\right\}$ since then neither $\left\{a_{1}, a_{3}\right\}$ nor $\left\{a_{2}, a_{3}\right\}$ divides $A$. Thus, $D_{1}=\left\{a_{1}, a_{3}\right\}$ and $D_{4}=\left\{a_{2}\right\}$. But now, $D_{2}$ and $D_{3}$ also partition $\left\{a_{1}, a_{2}, a_{3}\right\}$. This is a contradiction since $\frac{1}{4}+\frac{1}{5} \neq \frac{1}{2}$. Therefore, $m_{i}=m_{j}$ for some $i \neq j$. This is only possible if $D_{i}$ and $D_{j}$ partition $\left\{a_{1}, a_{2}, a_{3}\right\}$. Thus, $m_{i}=m_{j}=4$, $D_{i}=\left\{a_{1}, a_{2}\right\}$, and $D_{j}=\left\{a_{3}\right\}$. Observe that at least one of $\left\{a_{1}, a_{3}\right\}$ or $\left\{a_{2}, a_{3}\right\}$ divides $A$, else $A$ has two barren separations. Thus, $a_{1}+a_{3}=\frac{1}{3} \sum A$ or $a_{2}+a_{3}=\frac{1}{3} \sum A$. So, either $a_{1}=\frac{1}{12} \sum A$ or $a_{2}=\frac{1}{12} \sum A$. It must be that $a_{1}=\frac{1}{12} \sum A$, since $a_{2} \geqslant \frac{1}{6} \sum A$. Finally, $a_{2}=\frac{1}{6} \sum A$, since $a_{1}+a_{2}=\frac{1}{4} \sum A$. Thus, $A:=\{a, 2 a, 3 a, 6 a\}$ for some $a \in \mathbb{N}$, 
as required.

We may hence assume that every separation of $A$ is a neutral separation. Thus, $B$ divides $A$ if and only if $\sum B<\frac{1}{2} \sum A$. In particular, $\left\{a_{n}\right\}$ divides $A$ and so $a_{n} \leqslant \frac{1}{3} \sum A$. Let $M$ be a maximal set (under inclusion) among all subsets of $A$ containing $a_{n}$ and with sum at most $\frac{1}{2} \sum A$. Note that $\left\{a_{n}\right\}$ is a candidate for $M$, so $M$ exists. Since $\sum M<$ $\frac{1}{2} \sum A$, it follows that $M$ divides $A$ and hence $\sum M \leqslant \frac{1}{3} \sum A$. Choose $a \notin M$ and consider $M \cup\{a\}$. By choice of $M$ we have $\sum(M \cup\{a\})>\frac{1}{2} \sum A$. Thus, $A \backslash(M \cup\{a\}) \leqslant \frac{1}{3} \sum A$, since $A \backslash(M \cup\{a\})$ divides $A$. But now, $a_{n}>a \geqslant \frac{1}{3} \sum A$, which is a contradiction.

Combining Lemma 14 with Lemma 10 we have the following summary.

Theorem 15. For all $n \neq 3, d(n)=2^{n-1}$ and the sets that achieve this bound are precisely the anti-pencils or $A:=\{a, 2 a, 3 a, 6 a\}$ for some $a \in \mathbb{N}$. For $n=3, d(3)=5$ and the sets that achieve this bound are $\{a, 2 a, 3 a\}$ for some $a \in \mathbb{N}$.

We now present the end of the story for $d(n, 2 n)$ as well.

Lemma 16. Let $n \geqslant 3$ and let $A$ be a set of $2 n$ positive integers. If $A$ has exactly $\frac{1}{2}\left(\begin{array}{c}2 n \\ n\end{array}\right)$ divisors of size $n$, then $A$ is an $n$-anti-pencil.

Proof. Let $n \geqslant 3$ and let $A$ be a set of $2 n$ positive integers with $\frac{1}{2}\left(\begin{array}{c}2 n \\ n\end{array}\right)$ divisors of size $n$. Suppose $A:=\left\{a_{1}, \ldots, a_{2 n}\right\}$ with $a_{1}<\cdots<a_{2 n}$.

We claim that $A$ does not contain any abundant strong separations. Suppose not. We first suppose $n=3$. By Lemma $6, A$ has a unique abundant strong separation $\left\{\left\{b_{1}, b_{2}, b_{3}\right\},\left\{c_{1}, c_{2}, c_{3}\right\}\right\}$ with elements labelled in increasing order. Note that $b_{1}<\frac{1}{6} \sum A$ and $c_{1}<\frac{1}{6} \sum A$. Thus, swapping $b_{1}$ and $c_{1}$ yields a barren separation. If $b_{2} \leqslant \frac{1}{6} \sum A$, then swapping $b_{2}$ and $c_{1}$ yields another barren separation; a contradiction. Thus, $b_{2}>\frac{1}{6} \sum A$. On the other hand, $b_{2}<\frac{1}{4} \sum A$ since $b_{2}<b_{3}$. By symmetry, we also have $\frac{1}{6} \sum A<c_{2}<$ $\frac{1}{4} \sum A$. Thus, swapping $b_{2}$ and $c_{2}$ yields another barren separation. So we may assume $n \geqslant 4$. Let $\{B, C\}$ be an abundant separation of $A$ with $a_{1} \in B$. Let $c_{1}$ and $c_{2}$ be the two smallest elements of $C$. Define

$$
\begin{gathered}
\phi_{1}(\{B, C\}):=\left\{\left(B \backslash\left\{a_{1}\right\}\right) \cup\left\{c_{1}\right\},\left(C \backslash\left\{c_{1}\right\}\right) \cup\left\{a_{1}\right\}\right\} \text { and } \\
\phi_{2}(\{B, C\}):=\left\{\left(B \backslash\left\{a_{1}\right\}\right) \cup\left\{c_{2}\right\},\left(C \backslash\left\{c_{2}\right\}\right) \cup\left\{a_{1}\right\}\right\} .
\end{gathered}
$$

Since $n \geqslant 4$, it follows that $a_{1}, c_{1}$ and $c_{2}$ are each less than $\frac{1}{6} \sum A$. Therefore, both $\phi_{1}$ and $\phi_{2}$ are injective maps from the set of abundant strong separations to the set of barren strong separations. Furthermore, by Lemma 6, the images of $\phi_{1}$ and $\phi_{2}$ are disjoint. Therefore $A$ contains more barren strong separations than abundant strong separations; a contradiction. Thus, $A$ has no abundant strong separations as claimed.

It follows that every strong separation of $A$ must be neutral. Thus, an $n$-subset $B$ divides $A$ if and only if $\sum B<\frac{1}{2} \sum A$. Consider $M:=\left\{a_{1}, \ldots, a_{n-1}, a_{2 n}\right\}$. Note that $\sum M \leqslant \frac{1}{2} \sum A$, else $A$ is an $n$-anti-pencil and we are done. Hence, in fact $\sum M \leqslant$ $\frac{1}{3} \sum A$. Now let $B_{1}, \ldots, B_{\ell}$ be a sequence of $n$-subsets of $A$ such that $B_{1}=M, B_{\ell}=$ $\left\{a_{n+1}, \ldots, a_{2 n}\right\}$, and for each $1<j \leqslant \ell, B_{j}$ is obtained from $B_{j-1}$ by replacing some 
element of $B_{j-1}$ by a larger element not in $B_{j-1}$. Let $k$ be the first index such that $\sum B_{k}>\frac{1}{2} \sum A$, and let $B_{k}=B_{k-1} \triangle\{c, d\}$ with $c<d$. Since $B_{j-1}$ and $A \backslash B_{j}$ both have sum less than $\frac{1}{2} \sum A$, they both must divide $A$. Therefore, $B_{j-1} \leqslant \frac{1}{3} \sum A$ and $A \backslash B_{j} \leqslant \frac{1}{3} \sum A$. It follows that $\sum A-d+c \leqslant \frac{2}{3} \sum A$. Thus, $a_{2 n}>d>\frac{1}{3} \sum A$, which is a contradiction since $a_{2 n} \in M$.

Combining Lemma 16 with Lemma 2 we have the following summary.

Theorem 17. For all $n \neq 2, d(n, 2 n)=\frac{1}{2}\left(\begin{array}{c}2 n \\ n\end{array}\right)$ and the sets of $2 n$ positive integers that achieve this bound are precisely the $n$-anti-pencils. For $n=2, d(2,4)=4$ and the sets that achieve this bound are $A=\{a, 5 a, 7 a, 11 a\}$ or $A=\{a, 11 a, 19 a, 29 a\}$ for some $a \in \mathbb{N}$.

\section{Continuous analogues and open problems}

If one considers subsets of real numbers instead of natural numbers, then the question of divisibility no longer makes sense. In this context, it is natural to instead ask about subsets with non-negative sum.

Let $A$ be a set of $n$ real numbers. Define $\mu(A)$ (respectively $\mu_{k}(A)$ ) to be the number of subsets (respectively $k$-subsets) $B$ of $A$ such that $\sum B \geqslant 0$. We then define $\mu_{\min }(n)$ (respectively $\left.\mu_{\max }(n)\right)$ to be the minimum (respectively maximum) of $\mu(A)$ over all sets $A$ of $n$ real numbers with $\sum A=0$. Similarly, we define $\mu_{\min }(k, n)$ (respectively $\left.\mu_{\max }(k, n)\right)$ to be the minimum (respectively maximum) of $\mu_{k}(A)$ over all sets $A$ of $n$ real numbers with $\sum A=0$.

It is easy to check that $\mu_{\min }(n)=2^{n-1}+1$ for all $n \geqslant 1$, and that $A=\{1, \ldots, n-$ $1\} \cup\left\{\frac{-(n)(n-1)}{2}\right\}$ achieves this bound (recall that $\sum \emptyset=0$ ).

On the other hand, the minimization problem for $\mu_{k}$ is non-trivial. Indeed, the following nice conjecture of Manickam, Miklós, and Singhi asserts that $\mu_{\min }(k, n) \geqslant\left(\begin{array}{l}n-1 \\ k-1\end{array}\right)$, for $n \geqslant 4 k$.

Conjecture 18 ([10], [11]). If $n$ and $k$ are positive integers with $n \geqslant 4 k$, and $A$ is a set of $n$ real numbers with $\sum A=0$, then the number of subsets of $A$ with non-negative sum is at least $\left(\begin{array}{l}n-1 \\ k-1\end{array}\right)$.

Note that by choosing $A$ with exactly one non-negative element, we do obtain $\left(\begin{array}{l}n-1 \\ k-1\end{array}\right)$ non-negative $k$-subsets. Such sets correspond to the extremal examples in the Erdös-KoRado theorem [7], so we will call them $k$-pencils.

The Manickam-Miklós-Singhi conjecture has recently received substantial attention. We refer the reader to Alon, Huang and Sudakov [3], Chowdhury [4], Frankl [8], and Pokrovskiy [12].

We now discuss $\mu_{\max }(n)$ and $\mu_{\max }(k, n)$, which can be viewed as continuous analogues of our functions $d(n)$ and $d(k, n)$.

It is easy to see that maximizing $\mu(n)$ is equivalent to maximizing the number of subsets of $A$ whose sum is exactly zero. For $n$ odd, this reduces to a conjecture of Erdös 
and Moser, see [6, 5]. Using the Hard Lefschetz theorem [9] from algebraic geometry, Stanley $[13,14]$ solved a (generalization) of the Erdős-Moser conjecture.

Thus, by [13, Corollary 5.1], we have the following summary for $\mu_{\max }(n)$.

Theorem 19. For $n=2 \ell, \mu_{\max }(n)$ is achieved by taking $A=\{-\ell, \ldots,-1\} \cup\{1, \ldots, \ell\}$. For $n=2 \ell+1, \mu_{\max }(n)$ is achieved by taking $A=\{-\ell, \ldots \ell\}$.

As far as we know, determining $\mu_{\max }(k, n)$ is a wide open problem, although similar questions have been considered. For example, one can define $\mu_{\max }^{\prime}(k, n)$ to be the maximum of $\mu(A)$ over all sets $A$ of $n$ real numbers such that $\sum A<0$ and $\sum B<0$ for all $B \subseteq A$ with $|B|>k$. Recently, Alon, Aydinian and Huang [2] proved that $\mu_{\max }^{\prime}(k, n)=\left(\begin{array}{c}n-1 \\ k-1\end{array}\right)+\cdots+\left(\begin{array}{c}n-1 \\ 0\end{array}\right)+1$, settling a question of Tsukerman.

Note that by choosing exactly one element of $A$ to be negative, we have the bound $\mu_{\max }(k, n) \geqslant\left(\begin{array}{c}n-1 \\ k\end{array}\right)$. We overload terminology and call such a set a $k$-anti-pencil. This construction is not optimal for $n=2 k$, but in the range of the Manickam-Miklós-Singhi conjecture, we conjecture that it is.

Conjecture 20. If $n \geqslant 4 k$, then $\mu_{\max }(k, n)=\left(\begin{array}{c}n-1 \\ k\end{array}\right)$.

One can also attempt to characterize the extremal examples for $\mu_{\min }(k, n)$ and for $\mu_{\max }(k, n)$. For example, Chowdhury [4] gives some values of $k$ and $n$ for which the extremal examples for $\mu_{\min }(k, n)$ are necessarily $k$-pencils. Thus, it would be quite interesting to determine for which $k$ and $n$, the extremal examples for minimizing $\mu(k, n)$ and maximizing $\mu(k, n)$ are necessarily 'dual' to one another.

Problem 21. Determine for which $k$ and $n$ the only extremal examples for $\mu_{\min }(k, n)$ and $\mu_{\max }(k, n)$ are $k$-pencils and $k$-anti-pencils, respectively.

We end by mentioning that determining $d(k, n)$ for $n \neq 2 k$ is also an open problem. Recall that our proof technique relies on the fact that $B$ and $A \backslash B$ are both possible divisors of $A$, which fails when $n \neq 2 k$. Nonetheless, we conjecture that for most values of $k$ and $n, d(k, n)=\left(\begin{array}{c}n-1 \\ k\end{array}\right)$. Note that this agrees with the conjectured value for $\mu_{\max }(k, n)$. However, in the divisibility setting, it is possible that $d(k, n)=\left(\begin{array}{c}n-1 \\ k\end{array}\right)$ for all but finitely many values.

Conjecture 22. For all but finitely values of $k$ and $n, d(k, n)=\left(\begin{array}{c}n-1 \\ k\end{array}\right)$.

\section{Acknowledgements}

We thank Alex Heinis for valuable discussions during the 2011 International Mathematical Olympiad in Amsterdam. We also thank Ameera Chowdhury and Gjergji Zaimi for providing useful references. 


\section{References}

[1] International Mathematical Olympiad Problems. http://www.imo-official.org/ problems. aspx.

[2] Noga Alon, Harout Aydinian, and Hao Huang. Maximizing the number of nonnegative subsets. http://arxiv.org/abs/1312.0248, 2013.

[3] Noga Alon, Hao Huang, and Benny Sudakov. Nonnegative $k$-sums, fractional covers, and probability of small deviations. J. Combin. Theory Ser. B, 102(3):784-796, 2012.

[4] Ameera Chowdhury. A note on the Manickam-Miklós-Singhi conjecture. Eur. J. Comb., 35:131-140, 2014.

[5] R. C. Entringer. Representation of $m$ as $\sum_{k=-n}^{n} \varepsilon_{k} k$. Canad. Math. Bull., 11:289-293, 1968.

[6] P. Erdős. Extremal problems in number theory. In Proc. Sympos. Pure Math., Vol. VIII, pages 181-189. Amer. Math. Soc., Providence, R.I., 1965.

[7] P. Erdős, Chao Ko, and R. Rado. Intersection theorems for systems of finite sets. Quart. J. Math. Oxford Ser. (2), 12:313-320, 1961.

[8] Peter Frankl. On the number of nonnegative sums. J. Combin. Theory Ser. B, 103(5):647-649, 2013.

[9] S. Lefschetz. L'analysis Situs Et la Géométrie Algébrique. Collection de monographies sur la théorie des fonctions. Gauthier-Villars et cie, 1924.

[10] N. Manickam and D. Miklós. On the number of nonnegative partial sums of a nonnegative sum. In Combinatorics (Eger, 1987), volume 52 of Colloq. Math. Soc. János Bolyai, pages 385-392. North-Holland, Amsterdam, 1988.

[11] N. Manickam and N. M. Singhi. First distribution invariants and EKR theorems. J. Combin. Theory Ser. A, 48(1):91-103, 1988.

[12] Alexey Pokrovskiy. A linear bound on the Manickam-Miklós-Singhi conjecture. http: //arxiv-web3. library. cornell.edu/abs/1308.2176, 2013.

[13] Richard P. Stanley. Weyl groups, the hard Lefschetz theorem, and the Sperner property. SIAM J. Algebraic Discrete Methods, 1(2):168-184, 1980.

[14] Richard P. Stanley. Some applications of algebra to combinatorics. Discrete Appl. Math., 34(1-3):241-277, 1991. Combinatorics and theoretical computer science (Washington, DC, 1989). 